\title{
Alveolar cleft closure with iliac bone graft: a case report
}

\author{
Tichvy Tammama, ${ }^{1 *}$ Endang Syamsudin, ${ }^{1}$ Fathurachman ${ }^{2}$
}

\section{Abstract}

Objective: The present article is to report a case of a patient with alveolar cleft that was treated with the application of iliac bone graft Methods: A nine year-old girl with maxillary alveolar cleft had labioplasty and palatoplasty. The cleft was closed using iliac bone graft and the result was the canine erupts on the graft site get a better alveolar arch and no more oronasal fistule. Alveolar cleft can be treated by the use of iliac bone graft.

Results: Based on the clinical and radiographic development of the patient, the treatment for overall seemed success with the obtain of maxillary arch continuity, provide bone that is available for permanent canine to erupt, optimal alar base reconstruction, the fistulae was eliminated, provide a better nasal alar cartilage support, get a stabil the maxillary segment for orthodontic treatment and obtain an ideal alveolar morphology.

Conclusion: The timing of alveolar bone grafting usually associated with the state of the developing of dentition. Post operative management is important to get a good result and to prevent any complications.

Keywords: Gnatoschizis, Alveolar cleft, lliac graft, Gnatoplasty.

Cite this Article: Tammama T, Syamsudin E, Fathurachman. 2017. Alveolar cleft closure with iliac bone graft: A case report. Journal of Dentomaxillofacial Science 2(1): 37-40. D01:10.15562/jdmfs.v2i1.457

'Department of Oral and Maxillofacial Surgery, Faculty of Dentistry, Padjadjaran University, Hasan Sadikin General Hospital, Bandung, Indonesia

${ }^{2}$ Department of Orthopaedic, Faculty of Medicine, Padjadjaran University, Hasan Sadikin General Hospital, Bandung, Indonesia
"Correspondence to: Tichvy Tammama, Department of Oral and Maxillofacial Surgery, Faculty of Dentistry, Padjadjaran University, Hasan Sadikin General Hospital tichvy@yahoo.com

Received 04 0ctober 2016

Revised 30 January 2017

Accepted 04 February 2017

Available online 01 April 2017

\section{Introduction}

The alveolar cleft or gnatoschizis is a bony defect that exists in $75 \%$ patients with cleft lip and palate. Alveolar bone grafting is a surgical procedure that has been accepted with the purpose of obtaining maxillary arch continuity, to provide available bone for permanent canine to erupt, to maximize alar base reconstruction, to eliminate labial and palatal fistulae, to provide nasal alar cartilage support, to stabilize the maxillary segment after orthodontic treatment, to provide a solid maxillary structure before orthognatic surgery and to provide substructure for dental implant insertion or other prosthetic rehabilitation. .,2,7 $^{\text {p }}$

There are several controversies about the timing of the alveolar bone graft but it is most often related to the development of the permanent maxillary canine. ${ }^{1}$ Most of the surgeon did the bone graft at eight to ten year-old, before the eruption of permanent maxillary canine which aims to obtain bony support for the canine to erupt and to support the lateral insicors. Some authors reported that approximately $60 \%$ of cleft patients may have malformation of the maxillary lateral incisor due to its position near the cleft site, so they suggested to close the alveolar cleft with graft earlier, at the age of 5-6 years in order to give any lateral incisor an opportunity to migrate into and erupt through the bone graft. ${ }^{2,3,5}$

Some researchers suggested bone graft at an earlier age, between one and two years. However this is still controversial because it is not supported by the data that prove the effectiveness on the resistance of the alveolar bone in adulthood or on maxillary growth. ${ }^{2,8,9}$

The timing of alveolar cleft bone grafting has been divided into primary and secondary stages. Primary bone grafting performed after lip repair but before repairing the palate..$^{10}$ Secondary grafting defined as early secondary stage done as the child reach 2-5 years old, early mixed dentition at 6-8 years old, late mixed dentition at 9-12 years old and it is called late secondary grafting if it is done when the age reaches $13 .{ }^{12}$

There are several sources of graft: autograft bone that is harvested from the same person, allograft bone that is harvested from another person and synthetic grafts that composed of calcium, silicon or aluminum. Bone grafts have two types: cancellous bone, that is commonly used for grafting nonunios or cavity defects because it is quickly remodeled and incorporated and cortical bone that is slower to turn over than cancellous bone which is used for structural defects. ${ }^{15}$

For autotransplantation of bone in cleft patients, several donor sites have been exploited: rib, tibia, skull, chin and iliac rest. All of these sources have been used successfully because they contain pure bone, cancellous and autogenous. ${ }^{3,10}$ However, the iliac crest is used to be the most suitable donor site, since it is easier to get an adequate amount of cancellous bone by percutaneus incision. ${ }^{2,3,10}$ 

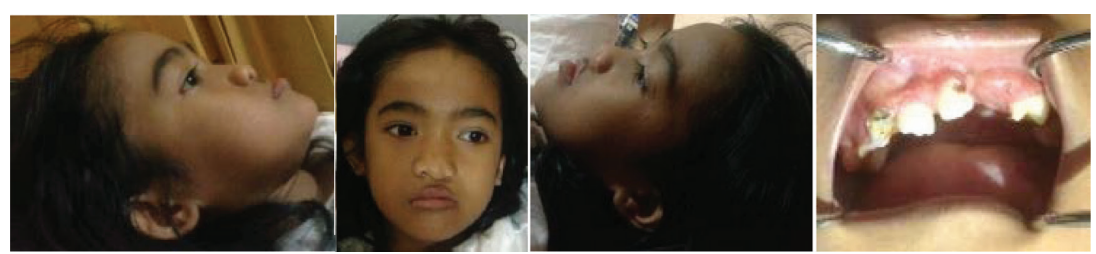

Figure 1 Extraoral and intraoral image of the patient before surgery

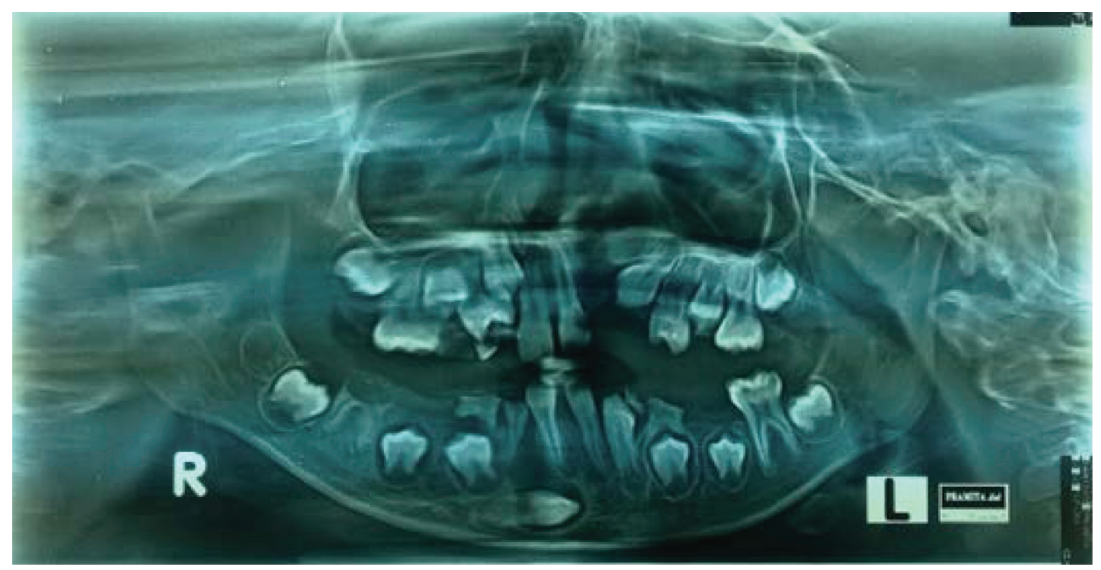

Figure 2 Panoramic radiograph before bone grafting
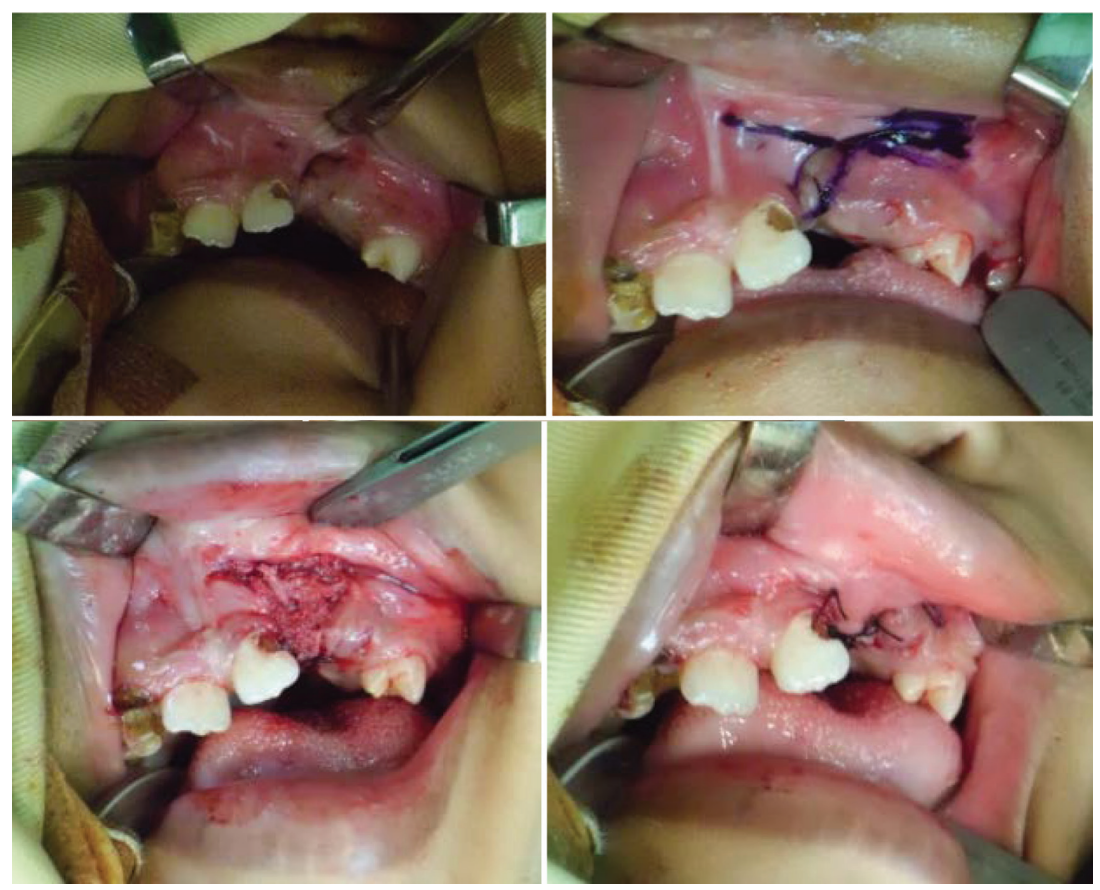

Figure 3 Alveolar cleft bone grafting sequence

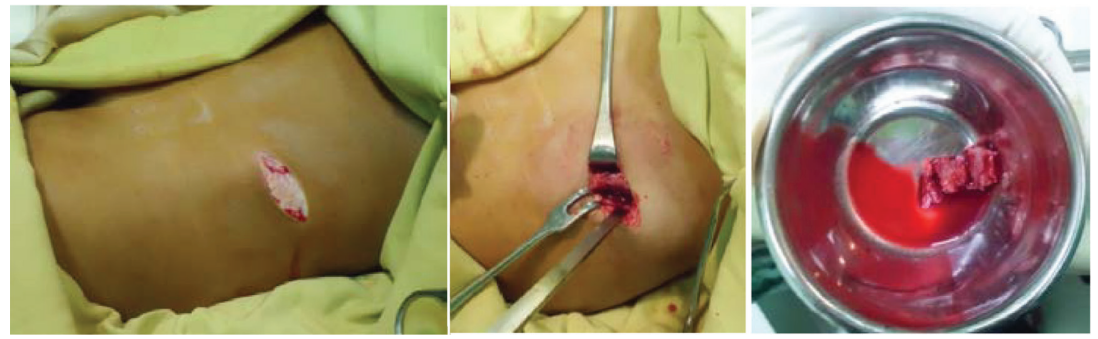

Figure 4 Autogenous iliac crest bone graft harvesting sequence

\section{Case Report}

A 9 year-old girl was admitted to Departement of Oral and Maxillofacial, Hasan Sadikin Hospital, Bandung with the chief complaint of alveolar cleft at 21-23 region, so when the patient was drinking, the water was often out from the nose. The patient was born with cleft of the lip and palate. When the patient was 7 months old, the cleft lip was repaired and at 4 years old, the cleft palate was repaired. Currently the patient came back again with the purpose to close the alveolar cleft. Panoramic radiography was performed before the procedure figure 1 and 2 .

The surgical procedures were performed under general anesthesia and local infiltration of pehacain $\mathrm{HCl}$ on the buccal and palatal aspect of the alveolar cleft. An incisional design was made along the gingival sulcus on the labial side extending into the cleft and palate. An incision was performed according to the design, make a full-thickness mucoperiosteal flap, separating the nasal mucosa from the gingiva. Closure of the nasal floor mucosa was performed with a simple interrupted suture.

The orthopedic harvested anterior iliac crest bone graft with a minimal skin incision of $2 \mathrm{~cm}$ long. An osteotome was then used to obtain a block of iliac bone of $1.5 \times 1 \times 0.5 \mathrm{~cm}$ as graft. The surgical site was irrigated, then the wound was closed with suture. The bone graft was then packed into the alveolar cleft in 21-23 area, the oral mucosa was closed with interrupted suture and the wound was covered by periodontal pack for 7 days figure 3 and 4.

For post-operative treatment, the patient was given antibiotic and analgesic. The patient was discharged a day after the surgery and was instructed to eat a soft diet for 1 week. The patient was also instructed to maintain oral hygiene with brushing her teeth using a soft brush and toothpaste a day after the surgery, and to do oral rinses with chlorhexidine after having meals for one week after surgery.

The patient was also instructed to return to clinic for a routine follow up visit. One week after surgery, the patient returned to the clinic with no complaint, the wound was cleaned with saline and the periodontal pack was removed figure 5. At the second week after surgery, the patient returned to the clinic, and the suture was removed. The canine was erupting at the graft site figure 6. Five months postoperatively, panoramic radiography examination was performed, for assessing and documenting quality of alveolar bone grafts figure 7 and 8, then the patient was referred to an orthodontic to repair the teeth occlusion. 


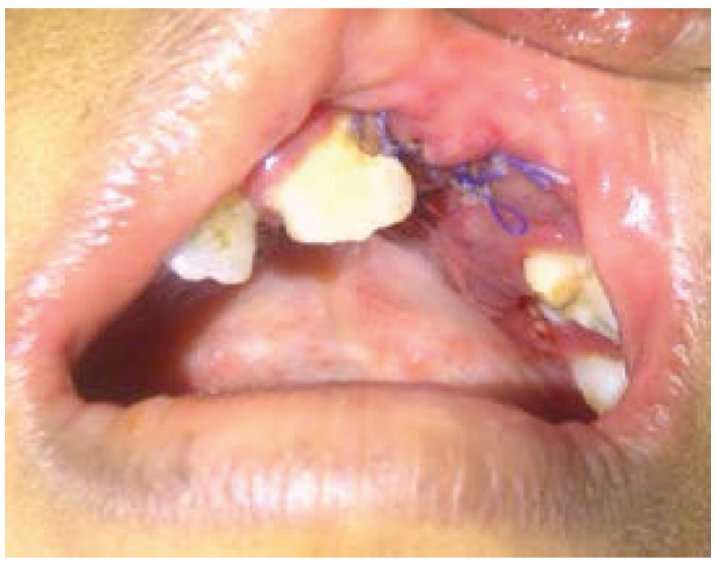

Figure 5 Intraoral image showed wound healing one week after bone grafting

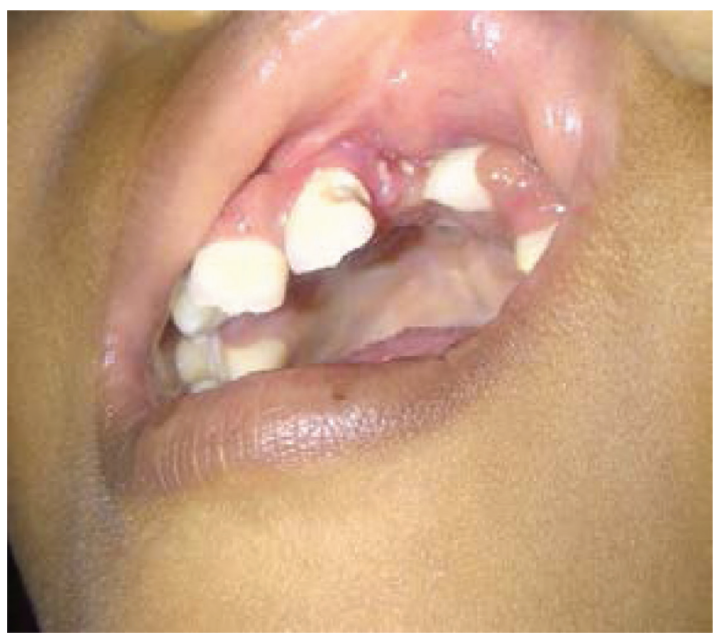

Figure 6 Intraoral image two weeks after bone grafting. The left maxillary canine was erupting to the graft site

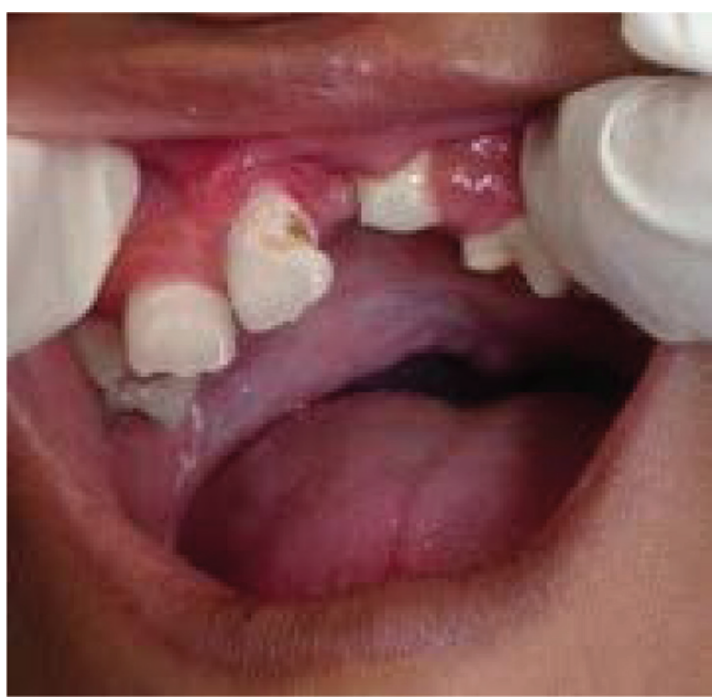

Figure 7 Intraoral image five months after bone grafting

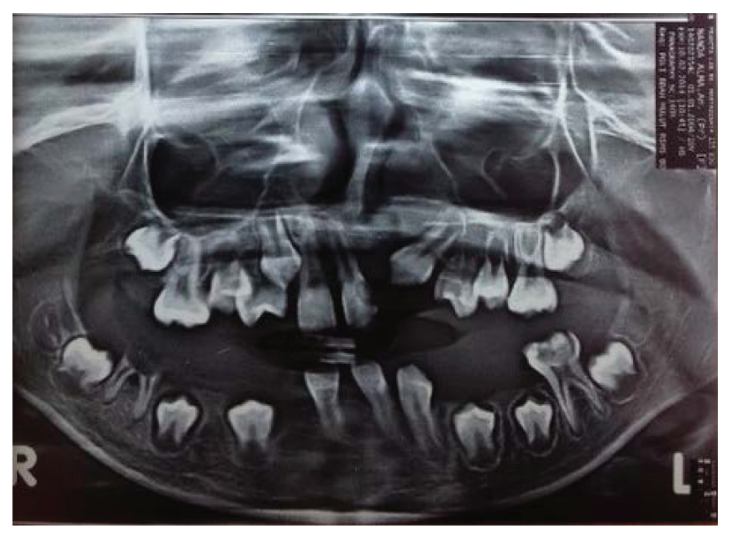

Figure 8 Panoramic radiograph five months after bone grafting

\section{Discussion}

The alveolar cleft is common in a patient with cleft lip and palate. The alveolar cleft requires bony repair to allow proper eruption of dentition in a normal alveolar arch or the teeth can be moved orthodontically into it. ${ }^{3,7,11}$ In the present case, the alveolar grafting was done at 9 year-old, before eruption of the permanent canines, in order to provide better bone support for the canine to erupt and to support the lateral insicors. ${ }^{11,12}$

At the present case, the alveolar cleft was closed using iliac bone graft, owing to its ease of access, and also it is easy to get an adequate amount of cancellous bone with a percutanous incision. ${ }^{2,3}$ Cancellous grafts subjected to a rapid revascularization that allow more rapid union and the osteoblasts lay down new bone on old trabeculae which are later remodeled, that is important for the longterm result. ${ }^{10,13}$ The autogenous graft may restore tooth-bearing function. ${ }^{1,14}$ Only autologous cancellous bone creates bone that responds normally to eruption and orthodontic movement of teeth. ${ }^{10}$ This bone is highly cellular, making it resistant to infection and optimal for rapid healing. ${ }^{4}$

Autogenous cancellous bone graft has four important properties: osteoconductive matrix, that acts as a scaffold or framework into which bone growth occurs; osteoinductive factors (growth factors such as bone morphogenetic protein (BMPs) and transforming growth factor beta (TGF- $\beta$ ) that promote local factors to stimulate bone formation); osteogenic cells (include primitive mesenchymal cells, osteoblasts, and osteocytes) and structural integrity. ${ }^{13}$

The concerns associated with iliac bone harvesting is the possibility of effects on growth, hematoma and donor site morbidity. Those complications could be minimized with a careful surgical technique with a minimally incision and stripping of the muscular attachment on the iliac 
crest, adequate hemostasis, careful wound closure and adequate postoperative pain control. ${ }^{1}$

The surgery technique was conservative with a minimally incision and without an agrresive dissection to the periosteum at the ilium to minimize complication at the donor site. ${ }^{1}$ Fresh autologous cancellous bone transforms very rapidly into alveolar bone. When autogenous cancellous bone is transplanted under optimal conditions, osteogenic cells in the graft will survive and new bone formation will start within a matter of days. ${ }^{10}$

Bone graft healing can be divided into five stages continuum: inflammation (chemotaxis activity stimulated by necrotic debris), osteoblast differentiation from precursors, osteoinduction (osteoblast and osteoclast function activity), osteoconduction (new bone forming over scaffold) and remodeling which process continues for years. ${ }^{13}$

Based on the clinical and radiographic development of the patient, the treatment for overall seemed success with the obtain of maxillary arch continuity, provide bone that is available for permanent canine to erupt, optimal alar base reconstruction, the fistulae was eliminated, provide a better nasal alar cartilage support, get a stabil the maxillary segment for orthodontic treatment, and obtain an ideal alveolar morphology.

\section{Conclusion}

In conclusion, the alveolar cleft closure using autogenous iliac crest for secondary grafting mostly used to obtain maxillary arch continuity, to provide available bone for permanent canine to erupt, to maximize alar base reconstruction, to eliminate labial and palatal fistulae, to provide nasal alar cartilage support, to stabilize the maxillary segment after orthodontic treatment, to provide a solid maxillary structure before orthognatic surgery and to provide substructure for dental implant insertion or other prostetic rehabilitation.

\section{Conflict of Interest}

The Authors report no conflict of interest

\section{References}

1. Cho-Lee G, Garcia-Diez E, Nunes R, et al. Review of secondary alveolar cleft repair. Ann Maxillofac Surg 2013;3: 46-50.

2. Bartlett S, Ehrenfeld M, Mast G, et al. Congenital Deformities. Unilateral CLP-Alveolar bone grafting; 2013.

3. Berkowitz S. Cleft lip and palate diagnosis and management. 2nd Edition. Germany; 2006.

4. Redett R. Alveolar bone grafts; 2013.

5. Yoshida S, Suga K, Nakano Y, et al. Postoperative evaluation of grafted bone in Alveolar cleft using threedimensional computed data. Cleft Palate Craniofac J 2013;50: 671-677.

6. Steinbacher DM, Padwa DL, Mulliken JB. Simultaneous harvesting of cancellous iliac bone for Alveolar cleft closure and dermis for augmentation median tubercle. Cleft Palate Craniofac J 2009;46: 295-298.

7. Goudy S, Lott D, Burton R, et al. Secondary Alveolar bone grafting outcomes, revisions, and new applications. Cleft Palate Craniofac J 2009;46: 610-612.

8. Fish J, Orth D. The clinical management of Postalveolar cleft palate infants. J Dent 1974;2: 239-146.

9. Akin Y, Ulgen O, Gencosmanoglu R, et al. Early Cheiloplasty. Its effects on Alveolar cleft. Eur J Plast Surg 1991;14: 164-167.

10. Eppley BL, Sadove AM. Management of Alveolar cleft bone grafting state of the art. Cleft Palate Craniofac J 2003;37: 229-233.

11. Miller LL, Kauffmann D, John SD, et al. Retrospective review of 99 patients with secondary Alveolar cleft repair. J Oral Maxillofac Surg 2010;68: 1283-1289.

12. Kazemi A, Stearns JW, Fonseca RJ. Secondary grafting in the Alveolar cleft patient. Oral Maxillofac Surg Clin North Am 2002;14: 477-490.

13. Brinker MR, Miller MD. Fundamentals of Orthopaedics. Philadelphia: WB Saunders; 1999. p. 7.

14. Balaji SM. Alveolar cleft defect closure with iliac bone graft, rhBMP-2 and rhBMP-2 with Zygoma Shavings: comparative study. Ann Maxillofac Surg 2011;1: 8-13.

15. Delee, Drez, Miller. DeLee and Drez's Orthopaedic sports medicine. Principles and practice. 3rd ed. Saunders Elsevier.

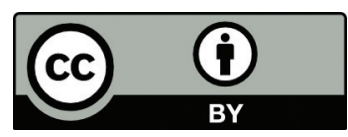

This work is licensed under a Creative Commons Attribution 